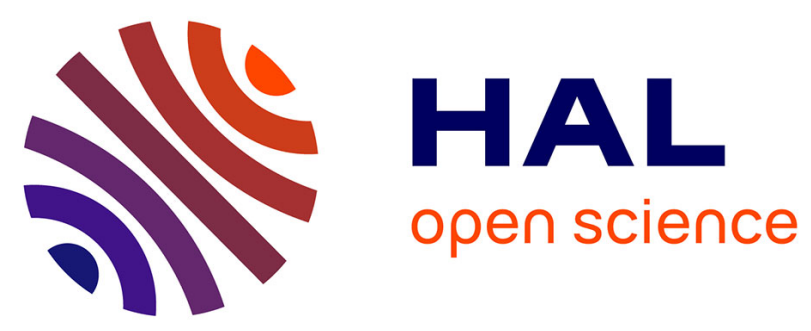

\title{
Performance Evaluation of Joint Transmission Coordinated-MultiPoint in Dense Very High Throughput WLANs Scenario
}

\author{
Iyad Lahsen Cherif, Lynda Zitoune, Véronique Vèque
}

\section{- To cite this version:}

Iyad Lahsen Cherif, Lynda Zitoune, Véronique Vèque. Performance Evaluation of Joint Transmission Coordinated-MultiPoint in Dense Very High Throughput WLANs Scenario. IEEE 40th Conference on Local Computer Networks (LCN 2015), Oct 2015, Clearwater Beach, FL, United States. pp.426-429, 10.1109/LCN.2015.7366344 . hal-01207074

\section{HAL Id: hal-01207074 \\ https://hal.science/hal-01207074}

Submitted on 5 Oct 2020

HAL is a multi-disciplinary open access archive for the deposit and dissemination of scientific research documents, whether they are published or not. The documents may come from teaching and research institutions in France or abroad, or from public or private research centers.
L'archive ouverte pluridisciplinaire HAL, est destinée au dépôt et à la diffusion de documents scientifiques de niveau recherche, publiés ou non, émanant des établissements d'enseignement et de recherche français ou étrangers, des laboratoires publics ou privés. 


\title{
Performance Evaluation of Joint Transmission Coordinated Multipoint approach in Dense Very High Throughput WLANs scenario
}

\author{
Iyad Lahsen Cherif*, Lynda Zitoune ${ }^{*}{ }^{\dagger}$ and Véronique Vèque * \\ * Signals and Systems Laboratory, Centrale-Supelec UPSUD CNRS \\ 3, Rue Joliot-Curie, 91192 Gif sur Yvette, France. \\ Email: iyad.lahsen-cherif, veronique.veque@1ss.supelec.fr \\ ${ }^{\dagger}$ Department of Systems Engineering, ESIEE-Paris \\ 2, boulevard Blaise Pascal, 93162 Noisy-le-Grand, France. \\ Email: lynda.zitoune@esiee.fr
}

\begin{abstract}
In this paper, we propose to use the Joint Transmission approach of Coordination Multipoint (JT-CoMP) of cellular networks, in order to reduce the interference impact in dense Very High Throughput (VHT) WLANs. VHT WLANs are based on wider channel bandwidth, efficient modulation techniques and support for spatial streams using MIMO schemes. However, the interference problem persists despite these approaches, and thereby prevents mobile stations to fully reaping the capacity improvement of such networks. We consider the stadium dense scenario, where the AP locations are carefully planned to optimize coverage and minimize cell overlap. To study the performance in such deployments, we develop a geometric stochastic model and investigate the benefit of JT-CoMP. Using simulation, we characterize the performance in terms of coverage and throughput for different sizes of coordinated set and carrier sensing domain of access points. Our results show that JT-COMP is an interesting technique for dense networks.
\end{abstract}

\section{INTRODUCTION}

In the last Wi-Fi standards and amendments, Task Groups of IEEE 802.11 are seeking to provide very high throughput (VHT) and low latency services over wireless LANs networks (WLANs). The aim is to fulfill the increasing demand of users and the exponential growth of wireless data traffic. As a consequence, critical applications like high definition video streaming (HDTV) and networked gaming can fully benefit from this new generation of WLANs. New standards like 802.11ac also called Gigabit Wi-Fi, are expected to provide up to $7 \mathrm{Gbps}$ in $5 \mathrm{GHz}$ band [13], [14], [5]. These improvements are based on three factors: wider channel bandwidth, efficient modulation techniques and support for spatial streams using MIMO scheme and its variations: Single User (SU-MIMO) in 802.11n, Multiple User (MU-MIMO) in 802.11ac [13], [14], [5]. However, even using these new generation of wireless access points (APs), the interference problem cannot be totally avoided in very dense networks.

High-density wireless networks are typically considered to be environments where the wireless devices like access points are placed in a very closed proximity and the number of client devices and required application throughput exceed the available capacity of a traditional "coverage-oriented"
Wi-Fi network design. Dense networks scenarios were first considered in multi-apartment buildings where a lot of home APs are deployed in each flat in a chaotic manner [2]. [2] gives the examples of cities like Boston and San Diego, where a particular wireless AP has about 80 other APs deployed in close proximity. A second example of dense WLAN is the stadium or arena deployment to offer high capacity Internet access and to enhance spectators experience by providing new services such as real-time concessions, ticket-ordering and online gambling. In this scenario, a lot of APs are deployed to offer the maximum capacity and the AP locations are carefully planned to optimize coverage and minimize cell overlap. In both scenarios, high density of APs causes a high interference level inducing collision in MAC transmission and reducing the overall customer throughput.

This interference also called inter-BSS (Base Service Set) interference is inevitable in such situations even using $802.11 \mathrm{ac} / \mathrm{n}$ technologies. Obviously, 802.11ac or $\mathrm{n}$ (with beamforming option) access point which provides CSI (Channel State Information) can reduce interference by selecting accurately beams to serve clients. However, in dense configuration where frequency reuse or planning is difficult to control, this objective is seldom easy. The inter-BSS is mainly due to three factors : 1) the lack of non-overlapping channels when large channels are used $(40 \mathrm{MHz}$ for $802.11 \mathrm{n}, 40 \mathrm{MHZ}$, $80 \mathrm{MHz}$, and $160 \mathrm{MHz}$ in $802.11 \mathrm{ac}$ ), for example only one non-overlapping $40 \mathrm{MHz}$ exists for $802.11 \mathrm{n}$ in the $2.4 \mathrm{GHz}$ band [1]. 2) The use of heterogeneous channel widths causing overlapping between primary and secondary channels like in 802.11ac [22]. 3) The unplanned deployment where APs are deployed in chaotic manner with factory default parameters. The latter is common with legacy $802.11 \mathrm{a} / \mathrm{b} / \mathrm{g}$ dense WLANs.

Recent work [22] shows by experiments that interference in 802.11ac WLAN is one of the major throughput degradation problem to face, due to unplanned selection of primary channels and channel widths when for example $20 / 40 \mathrm{MHz}$ channel is used in secondary channels of another $80 \mathrm{MHz}$ link. It suggests to design an appropriate interference mitigation 
scheme for the success of 802.11ac. Furthermore, in [1], the authors state that the capacity improvements of $802.11 \mathrm{n}$ can be fully utilized only when clients distribution is properly planned. However in dense networks, it is very difficult to know a priori the client location or their load distribution for both indoor and outdoor APs deployment.

Recently, for cellular networks, new revolutionary approaches have been developed and are expected to improve the throughput at the cell edge besides reducing interference in 5G cellular networks of the Public Private Partnership in Horizon 2020. These approaches called Coordinated Multipoint (CoMP) induce cooperation between base stations (BSs) and ensure both spatial and spectral efficiency of MIMO schemes [8], [7], [16]. Coordinated MultiPoint approaches are based on a constructive exploitation of interference, where the interferers BSs in the neighborhood cooperate together to serve the same device. The coordinated BSs form a coordinated set and use either Channel State Information (CSI) or user data to cooperate. Mainly three coordination multipoint techniques have been proposed: Joint Transmission (JT), Coordinated Beamforming/Scheduling (CB/CS) or Dynamic Point Selection (DPS) [21].

In this paper, we propose to use the Joint Transmission of Coordination Multipoint approach (we use the term JT-CoMP throughout the paper) to reduce the interference problem in dense VHT WLANs. We provide a framework to investigate JT-CoMP in dense WLANs and use simulation to show the gain of using such solution. The mathematical model takes into account both the interference and the contention domain of CSMA/CA when defining the set of coordinated APs. As stated earlier, in stadium scenario, a large number of APs close together within a large but highly concentrated area are placed in such a way to ensure performance requirements like coverage and capacity in RF environment where APs are LOS to each other with huge interference problem. In fact, in such environments, classical Poisson point process model of stochastic geometry is not appropriate at all. To develop our mathematical model we use the $r-l$ square point process of [4] which seems to be more appropriate to model such a dense WLANs, where the positions of WLAN APs are correlated in order to ensure a minimum coverage. We derive the coverage probability and the throughput formulas when JT-CoMP is used and evaluate by simulation these two performance parameters depending on the size of the coordinated set, i.e. the number of cooperating APs among the neighboring APs of a typical user equipment UE, and the SINR ratio. Two strategies are considered. In the first one, only APs beyond the contention domain of the serving AP but in vicinity of the UE are used to send the same signal as the serving AP. The second strategy, bolder than the first one, consists in coordinating the APs in the contention domain of the serving AP. In standard $802.11 \mathrm{~b} / \mathrm{g}$, these APs remain silent thanks to the DCF (Distributed Coordination Function) access control. In this case, coordinated APs are closer to the typical $\mathrm{UE}$ and guarantee a best transmission gain. Moreover, we give a few hints on how to integrate JT-CoMP in a CSMA/CA network where only one AP transmits at each time, but the synchronization problem between coordinated APs is beyond the scope of the present work.

This paper is structured as follows. We first introduce in section II some background materials and some related work on coordinated multipoint (CoMP) techniques. In section III, we recall the definition of $r-l$ Square p.p. and derive the underlying analytical model of JT-CoMP. The simulation results are discussed in IV.In V, we propose some insight on how to integrate the coordination in the network. We conclude the paper in section VI and give some perspectives to this work.

\section{BACKGROUND MATERIALS}

\section{A. VHT dense WLANs problems and limitations}

By integrating a Wi-Fi access point into the Internet box provided by ISP (Internet Service Provider), individuals can easily deployed a private network to connect all the devices (computers, tablets, smartphones or printers) together and to give all them a shared gateway to the Internet. As every household has access to the Internet at home, and its private WLAN, it has led to the emergence of high density 802.11 networks in urban areas and enterprises. High density wireless networks, by design, face significant challenges due to increased interference resulting from the close proximity of co-channel cells. Two types of dense WLAN have been identified: 1) multiapartment buildings where a lot of home APs are deployed in each flat in a chaotic manner [2], like cities of Boston and San Diego, where a particular wireless AP has about 80 other APs deployed in close proximity. 2) stadiums, amusement parks or arenas deployment to offer high capacity Internet access and to enhance spectators experience. In the last scenario, a lot of APs are deployed to offer the maximum capacity and the AP locations are carefully planned to optimize coverage and minimize cell overlap. In both scenarios, high density of APs causes a high interference level inducing collision in MAC transmission and reducing the overall customer throughput. For the first case of dense WLANs, i.e. Wi-Fi networks for offices, public buildings, schools, hospitals, are typically designed to optimize the capacity with a fairly large coverage area for each AP. In second case, stadiums for example, have different requirements such as to cover a very large number of people and devices within a large but highly concentrated area where users equipment and APs are LOS to each other [15]. As a consequence, the main challenge to be considered is an increased RF interference due several factors : 1) very high number of APs in closed proximity, 2) the reduced number of non-overlapping channels mainly when large channel widths are used to increase the capacity in order to guarantee the QoS requirements for applications, 3) an unplanned selection of primary channels and channel widths when for example $20 / 40 \mathrm{MHz}$ channel is used by an AP in secondary channels of $80 \mathrm{MHz}$ channel of another AP.

In [1], the authors explain that the network planning should be considered from "the three C's" perspective (context, coverage, and capacity). They point out the need to consider the 
interference when using $40 \mathrm{MHz}$ channels in $802.11 \mathrm{n}$ and the complex site-specific dependencies of MIMO in the planning phase and APs placement.

Dense WLANs were addressed in previous works using both experiments [2], [6], [20] and modeling [11], [9] to investigate the impact of interference in $802.11 \mathrm{a} / \mathrm{b} / \mathrm{g}$. In such networks, the interference is related to several factors: 1) lack of coordination between neighbors 2) restrictions of many ISP contracts which lead to over-deployment of APs, 3) handoff time reduction to meet multimedia QoS. 4) reduced number of non-overlapping channels [6]. The authors of [6] show that the cumulative throughput is characterized by the number of interfering access points rather than the number of clients. In [2], [6], some power control, rate regulation algorithms are designed to reduce interference among neighboring APs. In [20] other control strategies are evaluated like channel and power allocation algorithms, load-balancing algorithms, clientdriven association versus infrastructure-selected association, and $5 \mathrm{GHz}$ band-steering.

The author of the paper [11] propose a modified version of Matèrn point process of stochastic geometry to consider the spatial distribution of nodes: APs and mobile stations (MSs), by representing the impact of CSMA/CA. The proposed model is used to address some planning problem to provide QoS with a reduced deployment cost, relying on the effect of interferences and MAC constraints. The QoS parameters are defined by the coverage and the average bandwidth of each client. Another work [9] is interested in comparing the deployment of different wireless technologies: dense Wi$\mathrm{Fi}$, pico-cellular network with frequency planning and multicell joint processing transmission system from the technoeconomic point of view to meet a critical level of demand [9]. Using random sequential packing process, they estimate the required AP density to meet an average traffic demand and use it as the performance parameter for such comparison.

\section{B. Coordinated Multipoint in cellular networks}

CoMP is a cooperation technique used to reduce interference and hence to increase the cellular network throughput. In a cellular network, this cooperation is performed between BSs by exchanging data in joint transmission mode (JTCoMP) or the channel information in the Coordinated Beamforming/Coordinated Scheduling (CB/CS-CoMP) mode. In JT, users receive multiple copies of the same data from different BSs in the coordinated set, and the signal received from BSs outside the coordinated set is seen as interference. Several works investigate the modeling and evaluation of CoMP approaches using stochastic geometry in order to derive the closed form of coverage probability and throughput. In [17], authors characterize the SINR distribution and discusses some practical design problems. It concludes that increasing the BS density while fixing the cooperation radius improves the SINR. Also, the benefit of cooperation, in terms of coverage, increases with the path loss exponent. The work of [12] considers a Poisson Point Process to evaluate the coverage probability of a heterogeneous network under different connec- tivity models. It concludes that the $n$-strongest BS connectivity model (the $n$ BSs with strongest received signal) is better than the $n$-nearest BSs one (the nearest BS from each tier). In other words, defining a coordination set based on the strongest received power is more efficient than a coordinated set based on the short distance. In $\mathrm{CB} / \mathrm{CS}$, only beamforming vectors are shared between coordinated BSs. The effect of non ideal backhaul network is studied in [18]. Limited feedback issues are discussed in [3]. It is proved in [21], that the CoMP-JT strategy offers better performance than the $\mathrm{CB} / \mathrm{CS}$ thanks to the improvement of the received signal power. All these works consider Poisson Point Process to model the BSs location.

\section{Related work}

As for cellular networks, coordination CoMP between WiFi APs requires an ideal backhaul network with high-speed capacity and low delay, as well as a strict synchronisation in order to ensure simultaneous/coherent transmissions improving the signal quality at the device side. Few works have considered coordinated multipoint transmission in WLANs networks until now. The proposition of IEEE 802.11HEW (High Efficiency WLAN) [10] deals with the feasibility of CoMP in HEW networks and gives directives on how to integrate coordination in such networks. It proposes a centralized architecture where one AP is designed as controller (AC) to coordinate transmission between adjacent APs. Five schemes are defined: 1) the time is divided into two categories: Coordinated TX/RX Intervals (CTRI) and Non-Coordinated TX/RX Intervals (NCTRI), the AC schedules these intervals according to the amount of stations (STAs) and their distribution. 2) Coordinated power allocation by the AC 3) Coordinated frequency band allocation as in fractional frequency reuse in LTE. 4) Joint dynamic allocation of frequency and power which is the combination of coordinated power allocation and coordinated frequency band allocation. 5) Joint Transmission based on joint precoding as SU-MIMO/MUMIMO of 802.11ac.

To address the strict synchronization requirement of COMP, the authors of [10] suggest to use CPRI/IEEE 1588 for time and frequency synchronization among neighbor coordinated APs. A testbed is deployed to measure the benefit of joint transmission with joint precoding. The AC selects one AP from a coordinated set for transmission on the downlink, depending on the channel condition and load balance. The throughput gain is considerable compared to that using CoMP in LTE, thanks to the collision decrease and transmission opportunity increase.

[23] argues that the CSI of feedback scheme in MIMO network leads to a serious overhead problem mainly in outdoor deployment with mobility. So, it considers an indoor 802.11ac network and investigates, by simulation and measurement on a testbed, the $\mathrm{CB} / \mathrm{CS}$ approach of CoMP and compares its performance to MIMO Interference Alignment (IA). The paper also studies the impact of the time varying channel and the overhead using the 802.11ac feedback scheme. The obtained results show that $\mathrm{CB} / \mathrm{CS}-\mathrm{CoMP}$ provides better results than 
IA approach in terms of throughput i.e. $27 \%$ for IA against $47 \%$ using CoMP with overhead about $2,5 \%$. The throughput is reduced in case of time varying channel to $16 \%$ and $45 \%$ respectively. At the other side, the author of [19] show that a robust synchronization is guaranteed in between APs in a multi-hop WLAN thanks to the information acquired by nodes through a standard carrier sensing mechanism. So that, they do not need explicit synchronization messages to maintain a synchronized slotted execution of transmission.

In this paper, we first consider the $r-l$ square point process to describe the position of the APs and derive the model of JT-CoMP mechanism. Next, we use simulations to evaluate the gain. In the next section, we introduce the $r-l$ square p.p and explain the modeling steps of the coverage probability and throughput of the JT-CoMP.

\section{SySTEM MODEL : INTER-CELL INTERFERENCE COORDINATION}

In the past, dense deployments of wireless networks consisted of campus-like environments, where interference was mitigated by carefully planning cell layout. To characterize the increase of interference resulting from the close proximity of co-channel APs in high-density WLANs, we model this problem with stochastic geometry where the APs and devices are distributed using a $2 \mathrm{D}$ point process.

\section{A. APs locations model in stadium}

We consider a typical dense scenario as the one deployed in stadium.

We approximate this network by a grid model as represented in figure 1(a), where the APs positions are distributed over the plan according to $r-l$ square point process (figure1(b)). Here, we briefly describe the $r-l$ square point process and give the modeling steps of JT-CoMP based on this process.

As we said, Poisson point process generates nodes independently in the plane, which leads to many problems such as uncovered regions or nodes very close to each other causing strong interference. To overcome those problems, a new point process, called $r-l$ Square point process has been proposed as a planning tool to extend the cellular coverage in rural or poorly-covered regions using multimode femtocells. It models the position of these femtocells to meet requirements like coverage, throughput and energy efficiency [4]. $r-l$ Square point process is built as follows: the plane $\left(\mathbb{R}^{2}\right)$ is divided into squares of the sizes $r \times r$. In each $r \times r$ square, a new sub-square of size $l \times l$ (with $0 \leq l \leq r$ ) is placed. A point is uniformly distributed in each sub-square. Those points represent the APs. When $l<r$, this process is a Hard Core point process, as the points can not lie at a distance less than $r-l$. Hence, this model imposes that two points in adjacent squares can not be too close to each other, which reflects the real deployment of nodes and overcomes problems of the Poisson point process. Mobile stations are set according to a Poisson p.p. in the plane. Illustration of this point process is given in figure 1(b).

In the following, we focus on the downlink and we evaluate the performance in terms of coverage probability, under

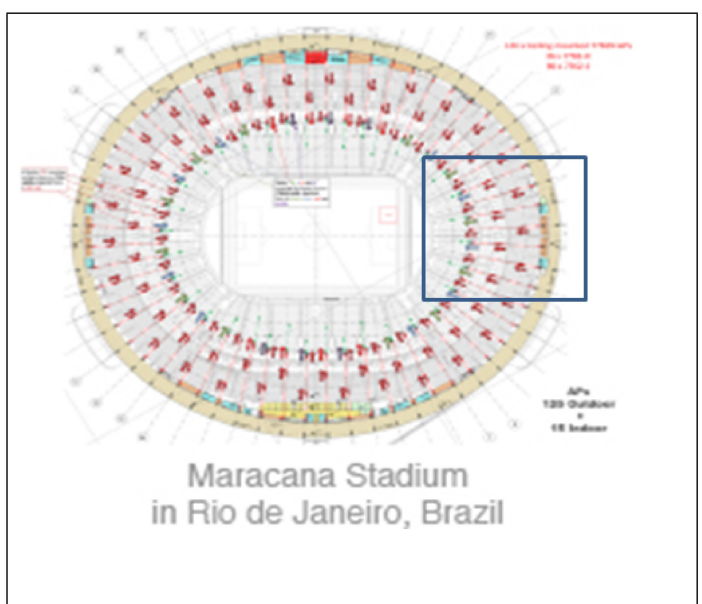

(a) Example of stadium dense WLANs deployment

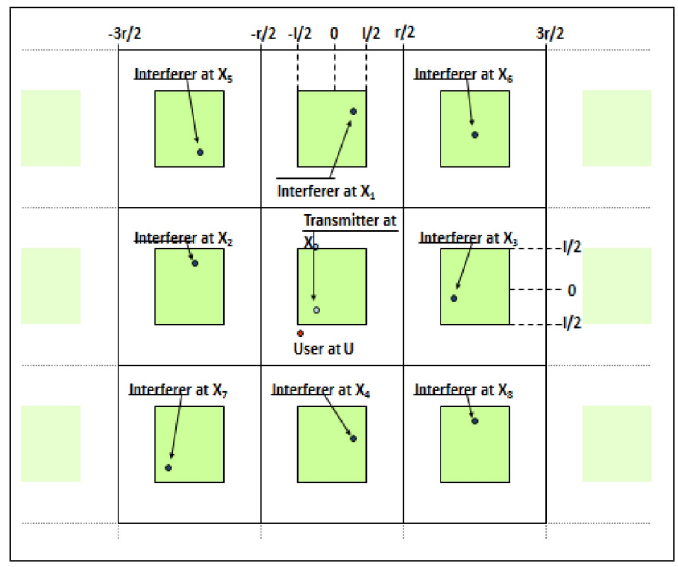

(b) r-1 square model approximation

Fig. 1. Stadium dense WLANs: approximation using $r-l$ square Point Process

the $r$ - $l$-Square model described above when coordination in particular JT-CoMP is applied between APs to mitigate the interference. We assume an ideal backhaul network relying APs to transmit all duplicated signals or to share data used for cooperation without collision and retransmission.

\section{B. Joint Transmission coordination model}

CoMP-JT is a cooperation technique used to reduce interference. In the classical CoMP-JT a mobile user receives data not only from the serving AP, but also from APs in its coordinated set. Signals received from APs outside the coordinated set are seen as interference. This is illustrated by equation (2). First, we define the coordinated set as follows:

$$
\mathcal{C}=\left\{X_{i} \in \Phi \text { s.t. } X_{i} \in B_{u}(d)\right\}
$$

where $\Phi$ is the $r-l$ Square p.p., $X_{i}$ is the $\mathrm{AP} i$ and $B_{u}(d)$ is the ball of radius $d$ centered at $u$, the typical user. Here $d$ : is the radius of the coordinated set.

Remark 1: As stated in cellular heterogeneous network [12], connecting to the $n$-strongest base stations enhance the performance better than the n-nearest base stations. In this 
work, since we suppose that all APs transmit at the same power level, the two strategies are equivalent.

The received signal at a typical user station $u$ is:

$$
y=\underbrace{\sum_{X_{i} \in \mathcal{A}} \sqrt{p_{t}} \xi_{i} \sqrt{l\left(\left\|X_{i}-u\right\|\right)}}_{\text {Useful signal }}+\underbrace{\sum_{X_{j} \in \mathcal{B}} \sqrt{p_{t}} \xi_{j} \sqrt{l\left(\left\|X_{j}-u\right\|\right)}}_{\text {Interference }}+\underbrace{N}_{\text {Noise }}
$$

where $p_{t}$ is the transmission power of nodes (APs), it is assumed to be the same for all APs, $\left(\xi_{i}\right)_{i}$ are i.i.d $\sim \mathcal{C N}(0,1)$ Gaussian random variables with mean 1 modeling fading, $l($.$) is the path loss function and N \sim \mathcal{C N}\left(0, \sigma^{2}\right)$ is an additive white Gaussian noise (AWGN). $\mathcal{A}$ and $\mathcal{B}$ represent the set of APs sending useful signals and the set of interferers, respectively.

The Distributed Contention Function (DCF) is a contentionbased decentralized protocol which uses Carrier Sense Multiple Access with Collision Avoidance (CSMA/CA). It allows to reduce collisions and enhance the network data rate.

Remark 2: In this paper, we are interested in one typical user, which represents all the remaining users in the network. Therefore, we derive the analytical expressions considering that only the serving AP (nearest to the mobile user) will perform the CSMA/CA procedure.

Let $C S_{t h r}$ be the carrier sensing threshold.

An AP can transmit if it satisfies the following carrier sensing condition:

$$
\mathbb{E}_{\xi}\left[\xi_{i}\left\|X_{i}\right\|^{-\alpha} p_{t}\right] \leq C S_{t h}
$$

Hence, an AP located at distance $\left\|X_{i}\right\|$ is allowed to transmit if and only if $\left\|X_{i}\right\| \geq\left(\frac{p_{t}}{C S_{t h}}\right)^{1 / \alpha}$. Let define the radius of carrier sensing domain as: $d_{C S}=\left(\frac{p_{t}}{C S_{t h}}\right)^{1 / \alpha}$.

We define the useful APs and the interferers sets as follows:

- $\mathcal{A}$ : is the set of APs in the coordination set $\mathcal{C}$ and outside the contention domain of the serving AP. It can be expressed as:

$$
\mathcal{A}=\left\{X_{i} \in \phi \text { s.t. } X_{i} \in B_{u}(d) \cap \bar{B}_{X^{*}}\left(d_{C S}\right)\right\}
$$

Where $X^{*}$ is the serving AP and $\bar{A}$ represents $\Phi \backslash A$.

- $\mathcal{B}$ : represents the set of interferers,

$$
\mathcal{B}=\left\{X_{i} \in \phi \text { s.t. } X_{i} \in \bar{B}_{u}(d) \cap \bar{B}_{X^{*}}\left(d_{C S}\right)\right\}
$$

Figure 2 illustrates the carrier sensing (CS) domain and the coordinated set $\mathcal{C}$.

Remark 3: Some of the silent APs which belong to the contention domain of CSMA/CA can also be used to coordinate the transmission to further enhance the network performance. The implementation of this strategy is more complicated than the used strategy in this paper as it requires to use another MAC access technique and a different channel should be used to avoid interference when coordinating APs outside the carrier sensing range.

In the following, we derive the analytical expression of the coverage probability of a typical user.

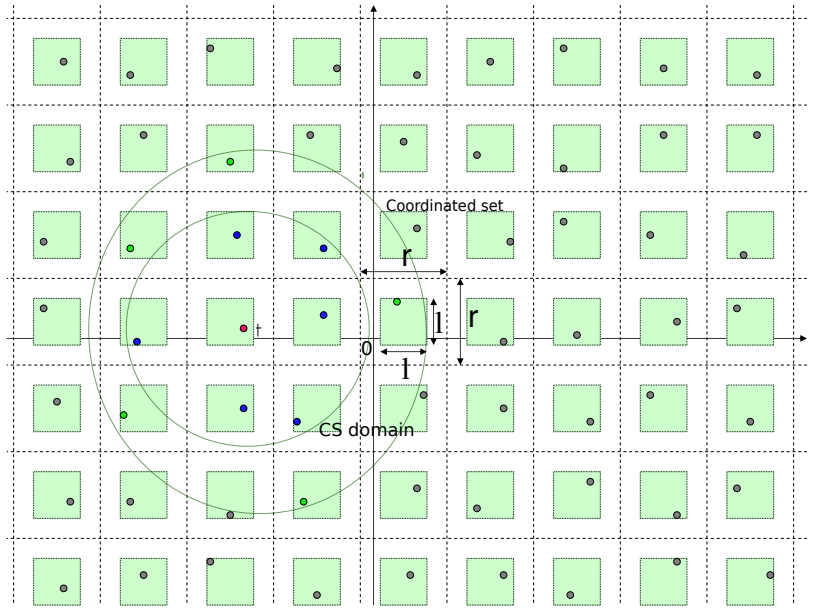

Fig. 2. The $r-l$ square p.p., the serving AP is the nearest one to the typical user, APs inside the CS domain are silenced by the CSMA/CA procedure and APs in $(\mathcal{A})$ are APs inside the coordinated set and outside the carrier sensing domain

Proposition 1: The coverage probability under JT-CoMP is given by:

$$
\begin{aligned}
p_{c}(T) & =\operatorname{Pr}(\operatorname{SINR}>T) \\
& =\prod_{j} \mathbb{E}\left[\left(\frac{1}{\left.\left.1+T \frac{l\left(\left\|X_{j}-u\right\|\right) \mathbb{1}_{\left\{X_{j} \in \bar{B}_{u}(d) \cap \bar{B}_{\left.X^{*}\left(d_{C S}\right)\right\}}\right.}}{\sum_{i} l\left(\left\|X_{i}-u\right\| \mid \| \mathbb{1}_{\left\{X_{i} \in B_{u}(d) \cap \bar{B}_{\left.X^{*}\left(d_{C S}\right)\right\}}\right.}\right.}\right)\right]}\right.\right. \\
& \times \mathbb{E}\left[\exp \left(-T \frac{\sigma^{2}}{\sum_{X_{i} \in \mathcal{A}} p_{t} l\left(\left\|X_{i}-u\right\|\right)}\right)\right]
\end{aligned}
$$

Proof:

$$
\begin{aligned}
p_{c}(T) & =\operatorname{Pr}(\operatorname{SINR}>T) \\
& =\operatorname{Pr}\left\{\frac{\left|\sum_{X_{i} \in \mathcal{A}} \sqrt{p_{t}} \xi_{i} \sqrt{l\left(\left\|X_{i}-u\right\|\right.}\right|^{2}}{\sum_{X_{j} \in \mathcal{B}} p_{t}\left|\xi_{j}\right|^{2} l\left(\left\|X_{j}-u\right\|\right)+\sigma^{2}}>T\right\} \\
& =\operatorname{Pr}\left\{\left|\sum_{X_{i} \in \mathcal{A}} \sqrt{p_{t}} \xi_{i} \sqrt{l\left(\left\|X_{i}-u\right\|\right)}\right|^{2}\right. \\
>T & \left.\left(\sum_{X_{j} \in \mathcal{B}} p_{t}\left|\xi_{j}\right|^{2} l\left(\left\|X_{j}-u\right\|\right)+\sigma^{2}\right)\right\} \\
& \stackrel{(a)}{=} \mathbb{E}\left[\exp \left(-T \frac{\sum_{X_{j} \in \mathcal{B}} p_{t}\left|\xi_{j}\right|^{2} l\left(\left\|X_{j}-u\right\|\right)+\sigma^{2}}{\sum_{X_{i} \in \mathcal{A}} p_{t} l\left(\left\|X_{i}-u\right\|\right)}\right)\right] \\
& =\mathbb{E}\left[\exp \left(-T \frac{\sum_{X_{j} \in \mathcal{B}} p_{t}\left|\xi_{j}\right|^{2} l\left(\left\|X_{j}-u\right\|\right)}{\sum_{X_{i} \in \mathcal{A}} p_{t} l\left(\left\|X_{i}-u\right\|\right)}\right)\right] \\
& \times \mathbb{E}\left[\exp \left(-T \frac{\sigma^{2}}{\sum_{X_{i} \in \mathcal{A}} p_{t} l\left(\left\|X_{i}-u\right\|\right)}\right)\right]
\end{aligned}
$$


where (a) follows the hyper exponential property. The first term is as follows:

$$
\begin{aligned}
& \mathbb{E}\left[\exp \left(-T \frac{\sum_{X_{j} \in \mathcal{B}} P_{t}\left|\xi_{j}\right|^{2} l\left(\left\|X_{j}-u\right\|\right)}{\sum_{X_{i} \in \mathcal{A}} P_{t} l\left(\left\|X_{i}-u\right\|\right)}\right)\right] \\
& =\mathbb{E}\left[\exp \left(-T \frac{\sum_{j}\left|\xi_{j}\right|^{2} l\left(\left\|X_{j}-u\right\|\right) \mathbb{1}_{\left\{X_{j} \in \bar{B}_{u}(d) \cap \bar{B}_{X^{*}}\left(d_{C S}\right)\right\}}}{\sum_{X_{i} \in \mathcal{A}} l\left(\left\|X_{i}-u\right\|\right)}\right)\right] \\
& =\mathbb{E}\left[\prod_{j} \exp \left(-T \frac{\left|\xi_{j}\right|^{2} l\left(\left\|X_{j}-u\right\|\right) \mathbb{1}_{\left\{X_{j} \in \bar{B}_{u}(d) \cap \bar{B}_{X^{*}}\left(d_{C S}\right)\right\}}}{\sum_{X_{i} \in \mathcal{A}} l\left(\left\|X_{i}-u\right\|\right)}\right)\right] \\
& \stackrel{(b)}{=} \mathbb{E}\left[\prod_{j}\left(\frac{1}{1+T \frac{l\left(\left\|X_{j}-u\right\|\right) \mathbb{1}_{\left\{X_{j} \in \bar{B}_{u}(d) \cap \bar{B}_{X^{*}}\left(d_{C S}\right)\right\}}}{\sum_{X_{i} \in \mathcal{A}} l\left(\left\|X_{i}-u\right\|\right)}}\right)\right] \\
& =\mathbb{E}\left[\prod_{j}\left(\frac{1}{1+T \frac{l\left(\| X_{j}-u||\right) \mathbb{1}_{\left\{X_{j} \in \bar{B}_{u}(d) \cap \bar{B}_{X^{*}}\left(d_{C S}\right)\right\}}}{\sum_{i} l\left(\| X_{i}-u||\right) \mathbb{1}_{\left\{X_{i} \in B_{u}(d) \cap \bar{B}_{X^{*}}\left(d_{C S}\right)\right\}}}}\right)\right] \\
& =\prod_{j} \mathbb{E}\left[\left(\frac{1}{1+T \frac{l\left(|| X_{j}-u||\right) \mathbb{1}_{\left\{X_{j} \in \bar{B}_{u}(d) \cap \bar{B}_{X^{*}}\left(d_{C S}\right)\right\}}}{\sum_{i} l\left(|| X_{i}-u||\right) \mathbb{1}_{\left\{X_{i} \in B_{u}(d) \cap \bar{B}_{X^{*}}\left(d_{C S}\right)\right\}}}}\right)\right]
\end{aligned}
$$

where (b) from the fact that $\left(\left|\xi_{i}\right|^{2}\right)_{i}$ are exponentially distributed.

Remark 4: In the Interference limited (free noise) regime, the coverage probability is independent from the transmit power of the LPNs.

The rate can be derived from the coverage probability by the following formula:

$$
\begin{aligned}
R & =\mathbb{E}\left(\log _{2}(1+\mathrm{SINR})\right) \\
& =\int_{0}^{\infty} \operatorname{Pr}\left\{\log _{2}(1+\mathrm{SINR}>t)\right\} d t \\
& =\int_{0}^{\infty} \frac{\operatorname{Pr}\left\{\operatorname{SINR}>e^{t}-1\right\}}{\log (2)} d t \\
& \stackrel{(a)}{=} \frac{1}{\log (2)} \int_{0}^{\infty} \frac{\operatorname{Pr}\{\operatorname{SINR}>x\}}{(x+1)} d x \\
& =\frac{1}{\log (2)} \int_{0}^{\infty} \frac{p_{c}(x)}{(x+1)} d x
\end{aligned}
$$

Where (a) follows from a variable change $\left(x=e^{t}-1\right)$.

Hence, the throughput is derived by replacing $p_{c}(T)$ (Eq. 7) in equation 22 .

Remark 5: The expectation in eq. 7 is over the p.p. and it is difficult to compute because the probability density function (pdf) of the $r$ - $l$ square p.p. is unknown. Hence, Monte Carlo simulation is used to validate results.

\section{Performance EVAluATION : SIMUlation AND RESULTS}

We consider a network composed of $7 \times 7$ (49) APs distributed in the plan according to the $r-l$ square p.p., all transmitting with the same power $p_{t}$. As we said, a typical user will have the same performance as the other users, so

\begin{tabular}{|c|c|}
\hline Parameters & Values \\
\hline$r$ & $50 \mathrm{~m}$ \\
\hline$l$ & $30 \mathrm{~m}$ \\
\hline Path-loss function & $l(d)=d^{-\alpha}$ \\
\hline$\alpha$ & 3.0 \\
\hline$p_{t}$ & $100 \mathrm{~mW}$ \\
\hline Bandwidth & $10 \mathrm{MHz}$ \\
\hline Noise & Normal $\left(1.0 e^{-11}, 3.76 e^{-11}\right)$ \\
\hline
\end{tabular}

TABLE I

PARAMETERS FOR THE NUMERICAL EVALUATION.

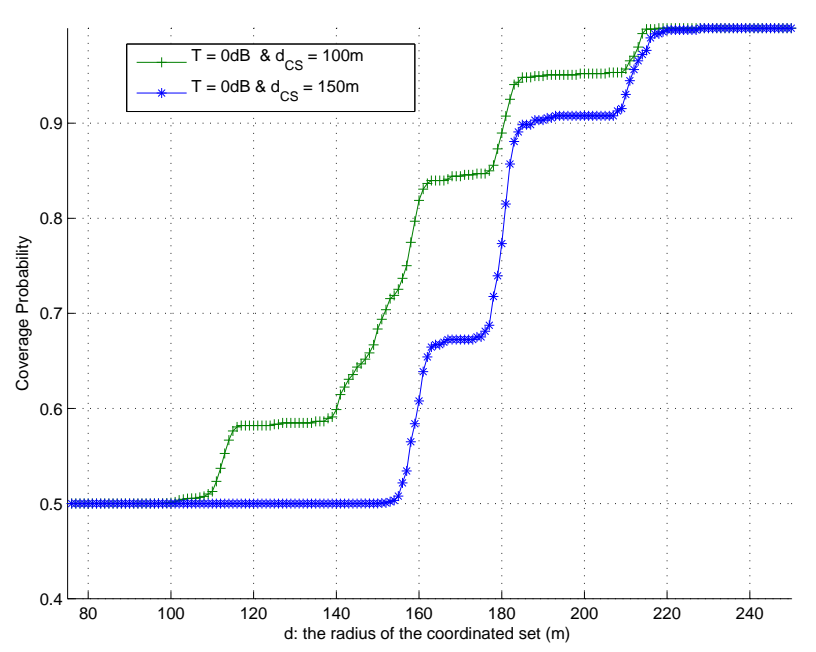

Fig. 3. The coverage probability vs d, the radius of the coordinated set for different values of the SINR threshold and for two values of $d_{C S}$ the carrier sensing radius

we do not derive results for all users. We consider one typical user placed uniformly at random in the grid. According to [9] which gives guidelines of APs characteristics, we set the transmission power to $p_{t}=100 \mathrm{~mW}(20 \mathrm{dBm}), r=50 \mathrm{~m}$, the sides of squares, and $l=30 \mathrm{~m}$, the sides of sub-squares. By this configuration, the distance between two nodes can not be less than $r-l=20 \mathrm{~m}$. The path loss model considered is given by: $l(r)=r^{-\alpha}$, where $\alpha$ is the path-loss exponent. Furthermore, we use a bandwidth of $10 \mathrm{MHz}$. We take, for analysis, the mean over 1000 realizations of the spatial process. Simulation parameters are summarized in table I.

Remark 6: As explained before, the carrier sensing domain and the cooperation domain, are illustrated by balls of radius $\mathrm{d}$ centered at $\mathrm{u}$ and $d_{C S}$ centered at $X^{*}$, respectively. To make comment of the result more clear and easier, and since de distance $\left\|u-X^{*}\right\|$ is not significant, we use the following nomenclature:

- $\mathrm{d} \leq d_{C S}$ : the coordinated set $\mathcal{C}$ is inside the carrier sensing domain, $B_{X^{*}}\left(d_{C S}\right)$.

- $\mathrm{d} \geq d_{C S}$ : the coordinated set includes the carrier sensing domain. 


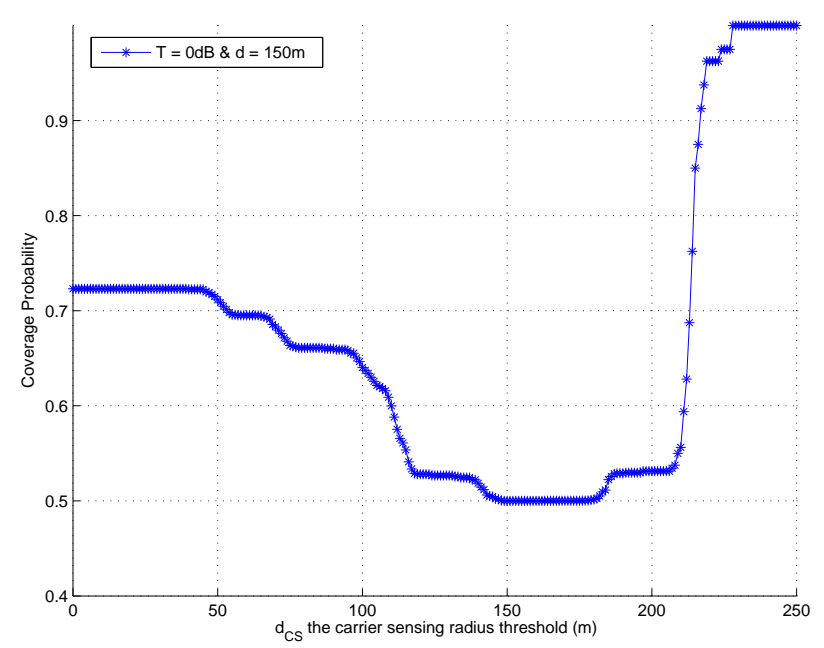

Fig. 4. The coverage probability vs $d_{C S}$, the radius of carrier sensing domain for $d=150 \mathrm{~m}$, radius of the coordinated set and for different values of the SINR threshold

In figure 3 , we plot the coverage probability versus the coordinated set radius for two values of the radius of the carrier sensing domain, $d_{C S}=100 \mathrm{~m}$ and $150 \mathrm{~m}$. It can be seen that the coverage probability remains constant $\left(p_{c}=0.5\right)$ for values of $\mathrm{d}$ less than $d_{C S}\left(\mathrm{~d} \leq d_{C S}\right)$ and increases for values of $\mathrm{d}$ $\geq d_{C S}$. This can be explained by the fact that by increasing $d$ more APs will join the coordinated set. However, since the serving AP performs the CSMA/CA procedure, the APs inside the ball of radius $d_{C S}$ are silent. Namely that an AP sends a useful signal if it is in the coordinated set and not in the coordination carrier sensing domain. The step function form of the increasing part of the coverage probability is explained as: $p_{c}$ remains constant until a new $\mathrm{AP}$ joins $\mathcal{A}$, then it moves to the next step.

In contrast, we keep the carrier sensing radius threshold fixed and we vary d. Figure 4 shows the coverage probability in function of the radius of the carrier sensing domain. It can be seen that the coverage probability decreases for values of $d_{C S}$ less than $\mathrm{d}$, the coordinated set $\mathcal{C}$ threshold, and increases for the values greater that $d$. This is because the number of potential coordinated APs muted (silent) grows when increasing $d_{C S}$ until it reaches $d$. Then, the coverage probability starts to increase, because interfering APs outside the coordination set and in the carrier sensing domain are silent.

Figure 5 shows the impact of the cooperation on the user rate. The radius of the carrier sensing domain is fixed to $d_{C S}=$ $150 \mathrm{~m}$. Remember that the maximal rate is about $195 \mathrm{Mbps}$. The rate remains constant for values of $\mathrm{d}$ less than $d_{C S}$. In fact, when increasing the radius of the coordination set, the APs in $\mathcal{C}$ become silent due to the CSMA/CA procedure. Starting form values of $\mathrm{d}$ around $d_{C S}$, the rate get improved. The reason is that the APs joining $\mathcal{C}$ are outside the carrier sensing domain. Hence, they send an effective signal which improves

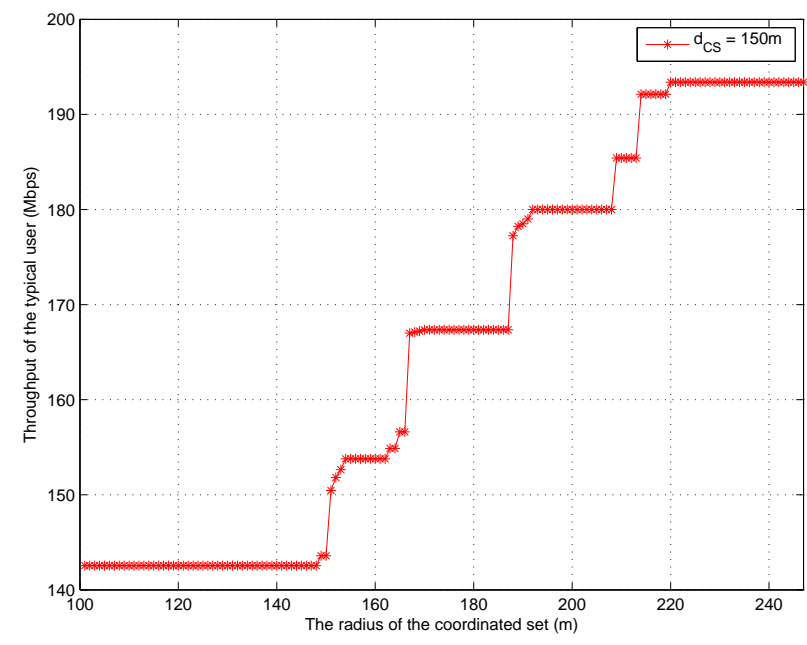

Fig. 5. The coverage probability vs $d_{C S}$, the radius of carrier sensing domain for $\mathrm{d}=150 \mathrm{~m}$, radius of the coordinated set and for different values of the SINR threshold

this performance metric.

\section{HOW TO IMPLEMENT JT-COMP IN CSMA/CA NETWORKS}

The main challenges to use CoMP approaches in WLAN are related to : 1) the use of CSMA/CA access control where only one AP transmits at each time; and 2) the lack of synchronization between WLAN APs when serving their user equipments [10]. The context is completely different from the cellular one where the BSs are synchronized in symbol and slot as in LTE network. Moreover, the position of BSs are planned unlike dense WLANs where the nodes location is mainly in ad hoc manner (in case of cities deployment). The paper [10] shows how to integrate coordinated transmission in Wi-Fi networks and show the throughput gain (by experience) using CoMP in 802.11HEW (High Efficiency WLAN).

To include JT-CoMP between APs of a WLAN, we propose to modify the control plan of 802.11 , mainly the association and re-association phase. When a node receives beacons from the neighboring APs, it first estimates the distance or the signal strength and next sends a request to ask for association with coordination. Also, during the transmission, APs in the coordinated set can use the Point Coordination Function (PCF) option and use the Contention Free Period (CFP) of the superframe to transmit without contention.

\section{CONCLUSION}

In this paper, we propose to use Joint Transmission approach of cellular networks to mitigate the interference problem in dense VHT WLANs. We provide a framework to model this approach using stochastic geometry and evaluate the performance by simulations, in terms of coverage and throughput.

Very High Throughput wireless LANs which use the latest IEEE $802.11 \mathrm{ac}$ or $802.11 \mathrm{n}$ standards, suffer from interference 
in dense configuration like stadium. Consequently, the mobile stations are prevent to fully reaping the capacity improvement of such networks. Usually, APs location deployed in a stadium are carefully planned to optimize coverage and minimize cell overlap. We use the $r-l$ square point process to model APs positions and derive the coverage probability and throughput when JT-CoMP is used.

By simulation, we evaluate the gain of JT-CoMP considering different values of SINR, the carrier sensing threshold of CSMA/CA access control and different sizes of the coordinated set. Our results show that JT-CoMP is a promising approach to use in dense wireless networks.

However, these results have to be confronted with operational implementation. Moreover, the proposed model can be used as a planning tool for coordination between APs by an operator considering real parameters of commercial APs in order to optimize the performance.

The main challenges to use JT-CoMP approaches in WLAN are related to the CSMA/CA medium access protocol which does not provide yet any synchronization between APs. In a future work, we will interest in how to integrate the JTCoMP coordination in WLANs according to [10], mainly by modifying the control plan of Wi-Fi networks, using ns3 simulator. Moreover, considering CoMP in dense WLANs with another access protocol like TDMA is an interesting perspective. Another extension of this work on improving the $r-l$ Square p.p. based on parameters related to the access protocol like Clear Channel Assessment (CCA) threshold.

\section{ACKNOWLEDGEMENT}

This work is part of the French project LCI4D of the Systematic Cluster, Paris Region Systems \& ICT Cluster (http://www.systematic-paris-region.org/).

\section{REFERENCES}

[1] "Deployment strategies for 802.11n: Key considerations for the next generation of wireless networking," Brocade Communications Systems, White paper, 2009.

[2] A. Akella, G. Judd, S. Seshan, and P. Steenkiste, "Self-management in chaotic wireless deployments," Wireless Networks, vol. 13, no. 6, pp. 737-755, December 2007.

[3] S. Akoum and R. W. H. Jr., "Interference coordination: Random clustering and adaptive limited feedback," CoRR, vol. abs/1210.6095, 2012.

[4] A. Busson, L. Zitoune, V. V. V., and B. Jabbari, "Outage analysis of integrated mesh lte femtocell networks," in In Proceedings of IEEE Global Communications Conference 2014, Austin, United States, December 2014.

[5] 802.11ac: The Fifth Generation of Wi-Fi, Cisco, August 2012, technical White Paper.

[6] M. A. Ergin, K. Ramachandran, and M. Gruteser, "Understanding the effect of access point density on wireless lan performance," in Proceedings of the 13th Annual ACM International Conference on Mobile Computing and Networking, ser. MobiCom '07. New York, NY, USA: ACM, 2007, pp. 350-353.

[7] R. Irmer, H. Droste, P. Marsch, S. Brueck, H.-P. Mayer, L. Thiele, and V. Jungnickel, "Coordinated multipoint: Concepts, performance, and field trial results," IEEE Communications Magazine, February 2011, iMT-Advanced and Next-Generation Mobile Networks.

[8] V. Jungnickel and al., "Field trials using coordinated multi-point transmission in the downlink," 21st International Symposium on Personal, Indoor and Mobile Radio Communications Workshops, 2010.
[9] D. H. Kang, K. W. Sung, and J. Zander, "Cost efficient high capacity indoor wireless access: Denser wi-fi or coordinated pico-cellular?" arXiv.org, Nov. 2012.

[10] Y. Li, Z. Luo, M. Yang, and B. Sun, "Feasibility of coordinated transmission for hew," September 2013, doc.: IEEE 802.11-13/1157r3.

[11] H. Nguyen, F. Baccelli, and D. Kofman, "A stochastic geometry analysis of dense ieee 802.11 networks," in INFOCOM 2007. 26th IEEE International Conference on Computer Communications. IEEE, May 2007, pp. 1199-1207.

[12] G. Nigam, P. Minero, and M. Haenggi, "Coordinated multipoint in heterogeneous networks: A stochastic geometry approach," in IEEE GLOBECOM Workshop on Emerging Technologies for LTE-Advanced and Beyond 4G (GLOBECOM-B4G'13), Atlanta, GA, December 2013.

[13] T. Paul and T. Ogunfunmi, "Wireless lan comes of age: Understanding the ieee 802.11n amendment," Circuits and Systems Magazine, IEEE, vol. 8, no. 1, pp. 28-54, First 2008.

[14] E. Perahia and M. X. Gong, "Gigabit wireless lans: An overview of ieee 802.11ac and 802.11ad," SIGMOBILE Mob. Comput. Commun. Rev., vol. 15, no. 3, pp. 23-33, Nov. 2011. [Online]. Available: http://doi.acm.org/10.1145/2073290.2073294

[15] Deploying Very High Density WiFi, Design and Configuration Guide for Stadiums, Ruckus Wireless, Inc, 2012, best practice design guide.

[16] S. Singh, A. Kumar, S. S. Khurmi, and T. Singh, "Coordinated multipoint (comp) reception and transmission for LTE-Advanced/4G," International Journal of Computer Science And Technology, vol. 3, June 2012.

[17] R. Tanbourgi, S. Singh, J. G. Andrews, and F. Jondral, "A tractable model for non-coherent joint-transmission base station cooperation," CoRR, vol. abs/1308.0041, 2013.

[18] P. Xia, C.-H. Liu, and J. G. Andrews, "Downlink coordinated multi-point with overhead modeling in heterogeneous cellular networks," CoRR, vol. abs/1210.5503, 2012.

[19] K. Xu, O. Dousse, and P. Thiran, "Self-synchronizing properties of csma wireless multi-hop networks," in SIGMETRICS, New York, June 14-18 2010, pp. 71-82.

[20] Y. e. a. Yiakoumis, "Behop: A testbed for dense wifi networks," in Proceedings of the 9th ACM International Workshop on Wireless Network Testbeds, Experimental Evaluation and Characterization, ser. WiNTECH'14, 2014, pp. 1-8.

[21] Y. Yifan, R. Yun, L. MingQi, S. Bin, and S. RongFang, "Achievable rates of coordinated multi-point transmission schemes under imperfect csi," In Proc. IEEE ICC, pp. 1-6, 2011.

[22] Y. Zeng, P. Pathak, and P. Mohapatra, "A first look at 802.11ac in action: Energy efficiency and interference characterization," in IFIP Networking Conference, 2014, June 2014, pp. 1-9.

[23] P. Zetterberg, "Interference alignment (ia) and coordinated multi-point (comp) overheads and rf impairments: Testbed results," in Vehicular Technology Conference (VTC Fall), 2014 IEEE 80th, Sept 2014, pp. $1-7$. 